\title{
Encoding Aerial Pursuit/Evasion Games with Fixed Wing Aircraft into a Nonlinear Model Predictive Tracking Controller
}

\author{
Jonathan Sprinkle, J. Mikael Eklund, H. Jin Kim and Shankar Sastry
}

\begin{abstract}
Unmanned Aerial Vehicles (UAVs) have shown themselves to be highly capable in intelligence gathering, as well as a possible future deployment platform for munitions. Currently UAVs are supervised or piloted remotely, meaning that their behavior is not autonomous throughout the flight. For uncontested missions this is a viable method; however, if confronted by an adversary, UAVs may be required to execute maneuvers faster than a remote pilot could perform them in order to evade being targeted. In this paper we give a description of a non-linear model predictive controller in which evasive maneuvers in three dimensions are encoded for a fixed wing UAV for the purposes of this pursuit/evasion game.
\end{abstract}

\section{INTRODUCTION}

The recent success of the Unmanned Aerial Vehicle (UAV) in gathering military intelligence [1] has invigorated research into UAV autonomy. Several reasons that a UAV is a viable alternative to a manned aircraft are its smaller size, reduced risk of loss of life, and smaller expense.

However, UAVs have thusfar been shown as a reliable and effective only against adversaries on the ground (e.g., avoiding small arms fire by flying at high altitudes). In order to be successful against an airborne adversary (either manned or unmanned) there are four possible dimensions in which to obtain an advantage: speed, maneuverability, munitions, and intelligence of control. Increasing the capability of the UAV in any of the first three categories will require either a redesign of the aircraft to increase its payload, maneuverability, engine size, or perhaps all three. Thus, the advantages of a UAV over manned aircraft (size, cost, etc.) are not as stark. However, by improving the intelligence of the aircraft, current aircraft designs may be reused with software changes.

Nonlinear model predictive control (NMPC) is promising as a control technique that explicitly addresses nonlinear systems with constraints on operation and performance. Aerial vehicles, with their nonlinear dynamics and input/state constraints to guarantee adherence to safe flight, are a proving ground for this

This research was supported under DARPA's IXO SEC program, under contract number DARPA SEC F33615-98-C-3614.

Department of Electrical Engineering and Computer Sciences, University of California, Berkeley. Berkeley, CA 94720, USA \{sprinkle, eklund, jin, sastry\}@eecs.berkeley.edu

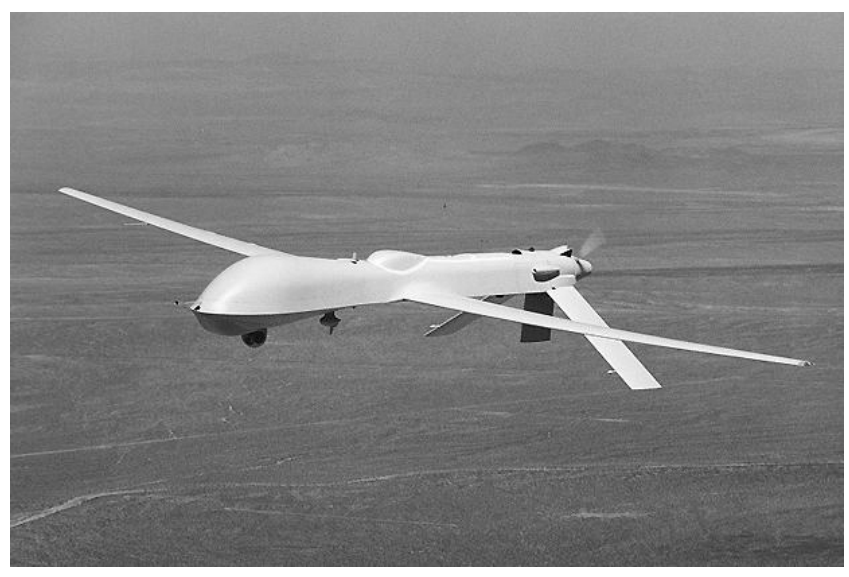

Fig. 1. The Predator Medium Altitude Long Endurance UAV (photo courtesy of US Department of Defense).

technology. In fact, in [2] the use of NMPC has been shown to be effective for rotary-wing UAVs. However, the use of these control methods that run in real-time on fixed-wing UAVs has not yet been shown. The need for 'fast' control algorithms for many dynamic systems has previously constricted the implementation of NMPC, since the algorithms must operate in real time.

In this paper, following the approach of [3], a numerically efficient nonlinear model predictive tracking control (NMPTC) algorithm is used to encode the pursuit/evasion game between two fixed-wing adversaries. The control problem is formulated as a cost minimization problem in the presence of input and state constraints. The minimization problem is solved with a gradient-descent method, which is computationally light and fast [3]. The NMPTC controller uses an interface to an existing autopilot in order to influence the system behavior. By formulating the cost function to include the state information of the other aircraft, input saturation, and state constraints, we show the performance of the NMPTC as a one-step solution for trajectory planning and control of UAVs competing in a pursuit/evasion game.

This paper details a NMPTC controller that is designed to provide evasive maneuvers of a fixed-wing UAV when confronted by an airborne adversary of $a$ priori type. Section II gives a brief description of the details of the fixed-wing aircraft used for flight and 
testing, and the expression of the UAV's dynamic and kinematic description. Section III describes the rules of the pursuit/evasion game, along with strategies for success by the pursuer/evader. Section IV gives a detailed description of the encoding of the pursuit/evasion game requirements into the controller, as well as implementation details for rapid simulation of the controller outside the provided testbed. Section $\mathrm{V}$ gives the results of some games using the controller, and Section VI presents our conclusions and continuing work.

\section{Vehicle Model}

\section{A. Aircraft details}

Due to restrictions on classified data and availability of aircraft, a Boeing T-33 two-place jet trainer will be used in our actual flight testing in June 2004, and will function as a UAV surrogate aircraft (called the UAV throughout this paper, although human passengers will be aboard for safety reasons). The route and trajectory of the UAV is controlled by an application running on a laptop PC that is interfaced to the avionics of the aircraft. The controller sends the control commands to the avionics pallet that transforms them into autopilot maneuver commands. The state of the UAV, as well as the state of the other aircraft (an F-15) is available via this avionics interface. The details of the avionics interface, the available state information, and the input controls are given in the rest of this subsection.

1) UAV testbed: In order to facilitate reliable testing, rapid integration, and a uniform interface independent of operating system, a CORBA-based platform was provided by Boeing to interface with the avionics of the UAV. This interface provides state information of the UAV, as well as the F-15, to an application that uses it. In addition, a high-level interface to the UAV autopilot is given that allows the interfacing application to control the rate of change of heading, rate of change of altitude, and (through interaction with the pilot) rate of change of velocity. Velocity changes by a controller are not available directly through the UAV auto-pilot, but instead an indicator alerts the pilot to increase/decrease thrust. As such, the timeliness of this input is not dependable during simulation.

The complex dynamics of the UAV are not captured in precise mathematical form, due to the imprecise knowledge of the autopilot's behavior with respect to the effect of input on state. In order to provide interfacing applications with a realistic idea of the behavior of the UAV with regard to certain inputs, Boeing also provided a "black box" simulation interface-known as DemoSim - that responds (in real-time) to autopilot commands. Using this DemoSim interface, it is possible for aircraft controllers to to test their behavior offline, and still have confidence in the results of those tests.
2) State Vector: The overall system state vector, $\mathbf{x}$, is defined using the following equations.

$$
\mathbf{x}=\left[\mathbf{x}^{K}, \mathbf{x}^{D}\right] \in \mathbb{R}^{n_{x}}
$$

The vector $\mathbf{x}$, which is the overall system dynamics, is partitioned in (1) into the kinematics (denoted by the superscript K) and system-specific dynamics (denoted by the superscript D) matrices. The kinematics of the system is given as the current state of the system in 3 dimensional space, and with respect to the 3 -axis posture of the body.

$$
\mathbf{x}^{K}=[x, y, z, \phi, \theta, \psi]
$$

The kinematics is shown in $(2)$, where $(x, y, z)$ is the position of the center of mass in 3 dimensions, $\phi$ is the roll, $\theta$ is the pitch, and $\psi$ is the yaw. The dynamics of the system is given as the time rate of change of the kinematic state variables, along with incidental changes, which are represented in classical notation as

$$
\mathbf{x}^{D}=[u, v, w, p, q, r],
$$

where $u=\dot{x}, v=\dot{y}, w=\dot{z}, p=\dot{\phi}, q=\dot{\theta}, r=\dot{\psi}$. Two state variables, the angle of attack and the angle of sideslip, are absent due to the lack of sensors available on the aircraft, and the autopilot's ability to guarantee heading and attitude of the aircraft.

3) Input vector: The input state vector, u, which is the space of possible inputs to the controller to modify the system state, is determined by the autopilot interface through which we have control of the system (as previously described). We define the input state vector as,

$$
\mathbf{u}=\left[u_{\dot{v}}, u_{\dot{\psi}}, u_{\dot{z}}\right] \in[-1,1]^{3} \in \mathbb{R}^{n_{u}}
$$

where $u_{\dot{v}}$ is the desired rate of change of airspeed velocity, $u_{\dot{\psi}}$ is the desired rate of change of turn, and $u_{\dot{z}}$ is the desired rate of change altitude. The input space is constrained by the $[-1,1]^{3}$ matrix. However, the actual values sent to the input controller are linearly mapped from the $[-1,1]$ range to the following ranges,

$$
\begin{aligned}
& \operatorname{map}\left(u_{\dot{v}}\right)= \begin{cases}-50[\mathrm{f} / \mathrm{s}] & -\infty<u_{\dot{v}}<-1 \\
{[-50,50][\mathrm{f} / \mathrm{s}]} & -1 \leqslant u_{\dot{v}} \leqslant 1 \\
50[\mathrm{f} / \mathrm{s}] & 1<u_{\dot{v}}<\infty\end{cases} \\
& \operatorname{map}\left(u_{\dot{\psi}}\right)= \begin{cases}-\pi / 50\left[\mathrm{~s}^{-1}\right] & -\infty<u_{\dot{\psi}}<-1 \\
{[-\pi / 50, \pi / 50]\left[\mathrm{s}^{-1}\right]} & -1 \leqslant u_{\dot{\psi}} \leqslant 1 \\
\pi / 50\left[\mathrm{~s}^{-1}\right] & 1<u_{\dot{\psi}}<\infty\end{cases} \\
& \operatorname{map}\left(u_{\dot{z}}\right)= \begin{cases}-10[\mathrm{ft} / \mathrm{s}] & -\infty<u_{\dot{z}}<-1 \\
{[-10,10][\mathrm{ft} / \mathrm{s}]} & -1 \leqslant u_{\dot{z}} \leqslant 1 \\
10[\mathrm{ft} / \mathrm{s}] & 1<u_{\dot{z}}<\infty\end{cases}
\end{aligned}
$$

where each of the mapped values are in the indicated units. The NMPTC controller will provide values in the $[-1,1]$ range, allowing the bounds of the mapping to 


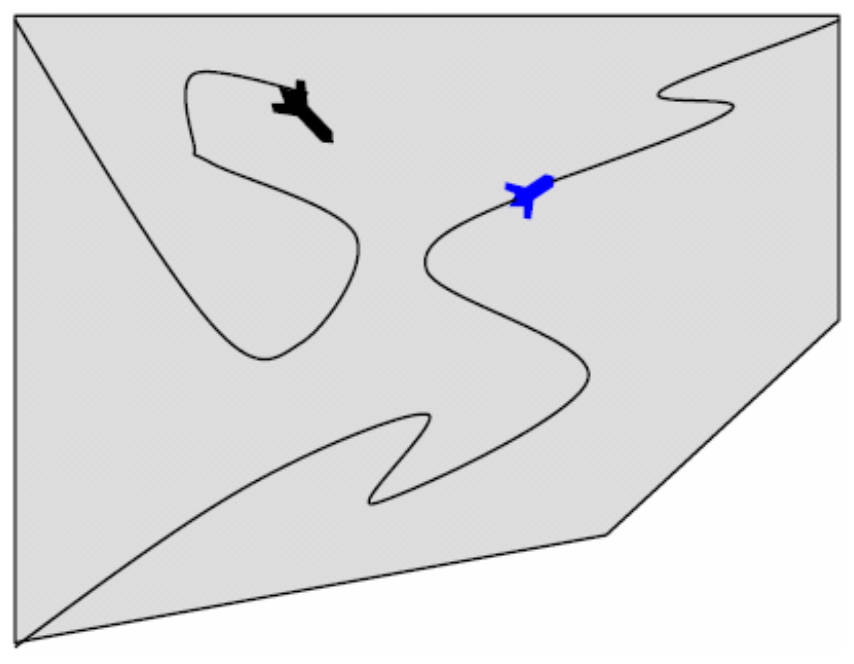

Fig. 2. An example run of the pursuit/evasion game.

be modified throughout simulation and testing of the controller to produce the desired results.

In addition, boundaries for the values of the state vector, $\mathbf{x}$, are integrated into the optimization cost function to prevent flight out of the test range, and to prevent violation of the minimum or maximum safe values for speed and altitude.

\section{The Pursuit/Evasion Game}

In the basic pursuit/evasion game (where the UAV plays the part of the evader, and the F-15 plays the part of the pursuer), there are asymmetric objectives for the pursuer and evader. The objective of the evader will be to either,

- fly for a predetermined period of time, $T$, since the start of the game; or

- exit the test range at an opposite corner without being targeted by the pursuer.

The objective of the pursuer will be to,

- target the evader before the end of the game.

The reason for the time limit, $T$ (20 minutes, for the purposes of this paper), is to prevent a trivial solution by the pursuer of haunting a position near the exit point to target the evader on exit. In our game, the pursuer can target the evader by aligning its heading with that of the evader and locating itself within a spherical cone (of predefined height, angle, and diameter) aligned with the tail of the evader. This cone, and a similar one for the pursuer, can be described by the angle off the tail (AOT) and angle off the nose (AON). The AOT is defined by

$$
\mathrm{AOT}=\cos ^{-1} \frac{A \bullet B}{|A||B|}
$$

where, $A$ is the directional vector of the pursuer's motion, and $B$ is the directional vector of the relative position of the evader with respect to the pursuer. AON is defined similarly with respect to the evader. AOT $=$ 0 corresponds to the pursuer being directly behind the evader, and $\mathrm{AON}=0$ corresponds to the evader being directly in front of the pursuer (the direction of flight of the former in each case is not considered).

The pursuit/evasion game [4], [5], [6] is an interesting application of NMPTC. As discussed in [7] there are four major types of strategies (or controls) for the pursuit/evasion game - open loop, state feedback, nonanticipative, and anticipative. An open loop strategy requires that each player decide their input signals without any knowledge of the other player's input vector. A state feedback strategy allows knowledge of each player's current input vector during the decision process. Nonanticipative strategies allow a player to use any input vector of the other player as long as the input vector does not correspond to a timestep in the future (with respect to the input being decided, not necessarily the current timestep). Finally, anticipative strategies place no restrictions on the current or future values of the input vectors being analyzed. Of course, it is important to note that the input vectors under consideration are only predicted inputs, and that they are subject to change if the other players use any of the four types of strategies.

\section{Controller Design for the Evader}

NMPC problems, in general, consist of the following steps; ${ }^{1}$. solve for the optimal control law starting from the state $\mathbf{x}(k)$ at time $k,{ }^{2}$ implement the optimal input $\mathbf{u}(k), \cdots, \mathbf{u}(k+\tau-1)$ for $1 \leq \tau \leq N,{ }^{3}$ repeat these two steps at time $k+\tau$.

The solution for the optimal control law can be found by formulating a cost function and considering it when performing the optimization. As described in [8], [2] it is possible to compose this cost function by using the specific details of the application, and the designers best knowledge of optimal performance of the object being tracked. Computational speed, and method, of this technique is discussed in detail in [2]. For our design, we chose the timestep $\tau=0.333[s]$, and a lookahead length of $N=30$ steps.

For this paper, we have included the design only of the evader, since our online controller will primarily be playing this role. However, we have also created a similar controller for the pursuer, for our own testing purposes.

In our application we choose to use an open-loop strategy for the pursuit/evasion game, since we have only state vector (and not input vector) knowledge of the other aircraft. We therefore encode the controller to use this strategy as a weighted member of its cost function. Taking into account the rules of the pursuit/evasion game we were able to design the evader controller by incorporating our desired outcome of the game. The desired trajectory of the evader, the location 
and orientation of the pursuer, the input constraints, and the state constraints, are each a part of the cost function. We set this cost function, $J$, to be

$$
J=\phi\left(\tilde{\mathbf{y}}_{N}\right)+\sum_{k=0}^{N-1} L(\mathbf{x}, \tilde{\mathbf{y}}, \mathbf{u}, \mathbf{d}),
$$

where,

$$
\phi\left(\tilde{\mathbf{y}}_{N}\right) \triangleq \frac{1}{2}\left(\tilde{\mathbf{y}}_{N}^{\mathrm{T}} P_{0} \tilde{\mathbf{y}}_{N}\right)
$$

and,

$$
\begin{aligned}
& L\left(\mathbf{x}_{k}, \tilde{\mathbf{y}}_{k}, \mathbf{u}_{k}, \mathbf{d}_{k}\right) \triangleq \\
& \quad \frac{1}{2}\left(\tilde{\mathbf{y}}_{k}^{\mathrm{T}} Q \tilde{\mathbf{y}}_{k}+\mathbf{x}_{k}^{\mathrm{T}} S \mathbf{x}_{k}+\mathbf{u}_{k}^{\mathrm{T}} R \mathbf{u}_{k}+\frac{1}{\left(\mathbf{d}_{k}^{\mathrm{T}} G \mathbf{d}_{k}\right)^{\frac{1}{4}}}\right)
\end{aligned}
$$

In these equations, $\mathbf{x}$ is the state vector, and $\mathbf{u}$ is the input vector. The vector $\tilde{\mathbf{y}}$ is the encoding of the error on the current trajectory, and is defined as $\tilde{\mathbf{y}} \triangleq$ $\mathbf{y}_{d}-\mathbf{y}$, where $\mathbf{y}=C \mathbf{x} \in \mathbb{R}^{n_{\mathbf{y}}}$. The vector $\mathbf{y}_{d}$ is the desired trajectory of the aircraft at the given timestep. The vector $\mathbf{d}$ is the proximity danger vector between the evader and its adversary.

The $Q, S, R, G$, and $P_{0}$ square matrices each serve as a balancing factor in the cost function. By modifying their relative values of each of these matrices, it is possible to give more "weight" to certain portions of the cost function. We chose to give values to these matrices so that in an equilibrium condition no single factor would outweigh the others, and the aircraft would continue at the same speed on its heading. For this controller ad hoc methods are used to find these weighting factors, which required the ability to perform simulations rapidly to reduce the time of development.

In the definition of $\tilde{\mathbf{y}}, C$ acts as a filter to remove elements in $\mathbf{x}$ that are unimportant to the rules of the game. The values for $Q$ differ from those in $S$, necessarily, as the $S$ matrix is used to ensure that the statically defined constraints on the state vector (e.g., maximum/minimum velocity) are not violated, while the $Q$ matrix is used to ensure that the state values important to winning the game are properly weighted.

The $\mathbf{d}$ vector is the difference in position and heading of the evader and the pursuer. It is used to calculate the proximity of the adversary, and figures into the cost function to outweigh the desired trajectory, should the adversary invade the safe region surrounding the evader. Note that $\mathbf{d}$ contains position information, as well as the angle off tail measurement, which describes the relative relationship of the position of the adversary (regardless of its heading) to the evader's tail. The $G$ matrix, then, is used to appropriately weight this component of the cost function.
The solution to the cost-function optimization (using the iterative technique described in [3], [9]) requires the calculation of the derivatives of the vehicle dynamics with respect to both the state and input vectors. Since mathematical equations were not available for the vehicle dynamics - only the DemoSim interface - we used a simplified definition using the Eulerian equations of motion to capture our "projected" values for the state of the evader (and pursuer) in the predictive component of the controller.

\section{A. Simulating in accelerated time}

The DemoSim environment - our authoritative model of the aircraft dynamics - provides a state vector response to inputs in real-time. This means that when using DemoSim with our controller, our operations occur at the same rate as they would on the actual aircraft. When configuring the relative weights of the cost-function elements, however, running simulations for 10 minutes at a time meant that we could make fewer than 6 changes per hour to the weights. As such, it was necessary to simulate in an accelerated timeframe to allow for rapid feedback when manipulating the matrix values to balance the cost function.

By using our simplified definition of the equations of motion, it was possible for us to approximate the behavior of the aircraft (first in two dimensions, and then in three) using our predictive representation. This allowed us to execute a simulation time of 10 minutes in less than 10 "wall-clock" seconds, thus increasing our changes per hour by a factor of 60 .

\section{B. Matching dynamics with DemoSim}

It was important, however, to ensure that our simplified dynamics matched the DemoSim dynamics within an acceptable margin of error. Confirmation that our simplified dynamics were reasonably close would enable us to make accurate decisions for matrix weighting during rapid simulation/resimulation phase, and would also show that our predictive model was reasonably close to how the aircraft would actually behave. By modifying the latency gains for the Eulerian equations, we were able to get a reasonably accurate dynamics model. Fig. 3 shows the aircraft behaviour predicted by our model and used by the NMPTC controller at various look-ahead ranges, versus the DemoSim model behaviour, for a simulation of time 3 minutes.

\section{Simulation Results}

\section{A. Implementation}

The NMPTC algorithm and the pursuit/evasion game were implemented in $\mathrm{C}++$ and run in a Windows environment. Two instances of the aircraft were simulated, one as the pursuer and one as the evader. For these examples, the performance characteristics of the two aircraft being identical, as will be the case in all the 

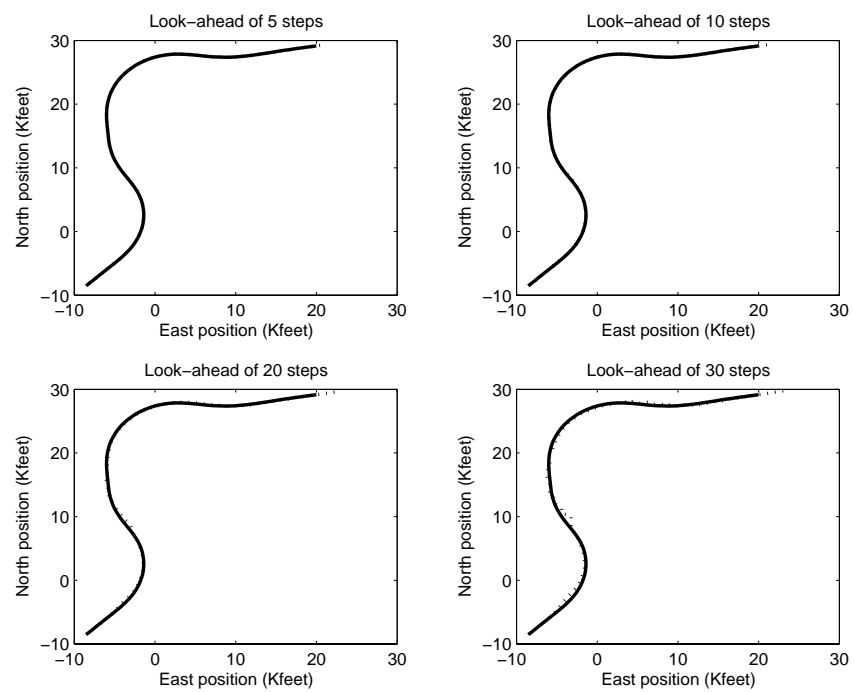

Fig. 3. Comparison between the predicted behaviour of the aircraft used by the controller (dashed line), and the DemoSim "actual" behaviour (solid line).

simulations leading up to the final test flight due to the constraints of the DemoSim interface.

The evader aircraft's NMPTC controller was tuned to provide for a successful game outcome in terms of exiting the playing area and avoiding the pursuer aircraft. The former criterion was encoded in the algorithm through the $Q$ matrix of (9), the latter in the $G$ matrix along with the choice of states that were included in the vector $\mathbf{d}$, as described above.

The pursuer aircraft was tuned to close with the evader using its $Q$ matrix and the choice of $\mathbf{y}_{d}$ as a path toward the predicted position of the evader. Both aircraft controllers used the $S$ and $R$ matrices to constrain the states and inputs.

\section{B. Results}

A typical pursuit/evasion game result is shown in Fig. 4. In this case the evader has entered in the southwest corner and it trying to exit from the northeast corner of the playing area. The pursuer has entered in the southeast corner and immediately begins to close with evader. In this result, the evader has chosen to turn in the pursuer's direction to avoid allowing the pursuer to take a shooting position on its tail. Turning and climbing/descending maneuvers follow during which the evader is still trying to proceed toward the egress (exit) point to the northeast until the game time expires after 20 minutes. A 2-dimensional view is also provided in Fig. 5 for better viewing of the aircraft flight paths.

In this game, the pursuer win condition was defined by the pursuer being in the $10^{\circ} \mathrm{AOT}$ cone of the evader (i.e. within $10^{\circ}$ of directly behind the evader) while at the same time having the evader within the $10^{\circ}$ angle off nose $(\mathrm{AON})$ cone of itself (i.e. having the evader within

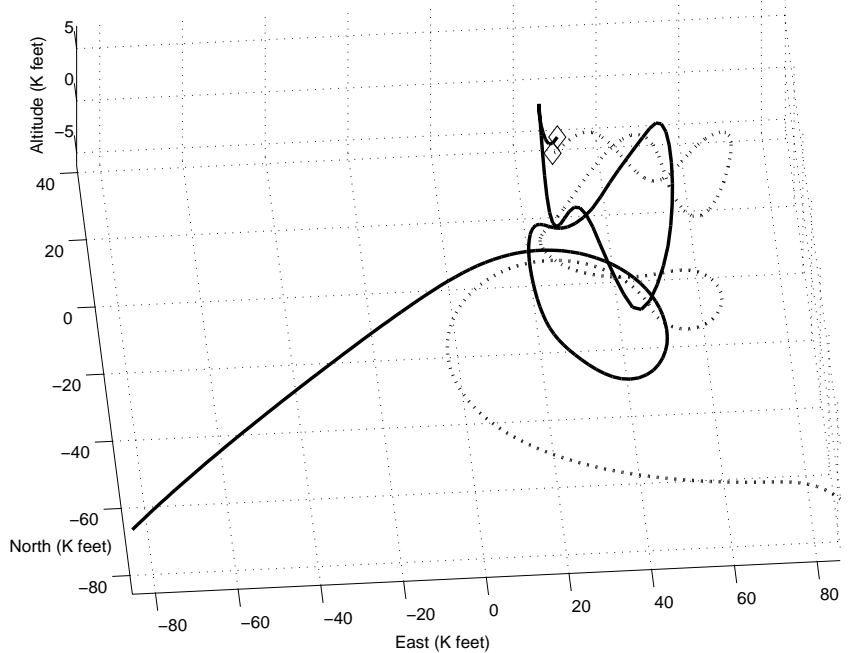

Fig. 4. An example run of the pursuit/evasion game viewed in three-dimensions. The evader path is solid, and the pursuer path is dotted.
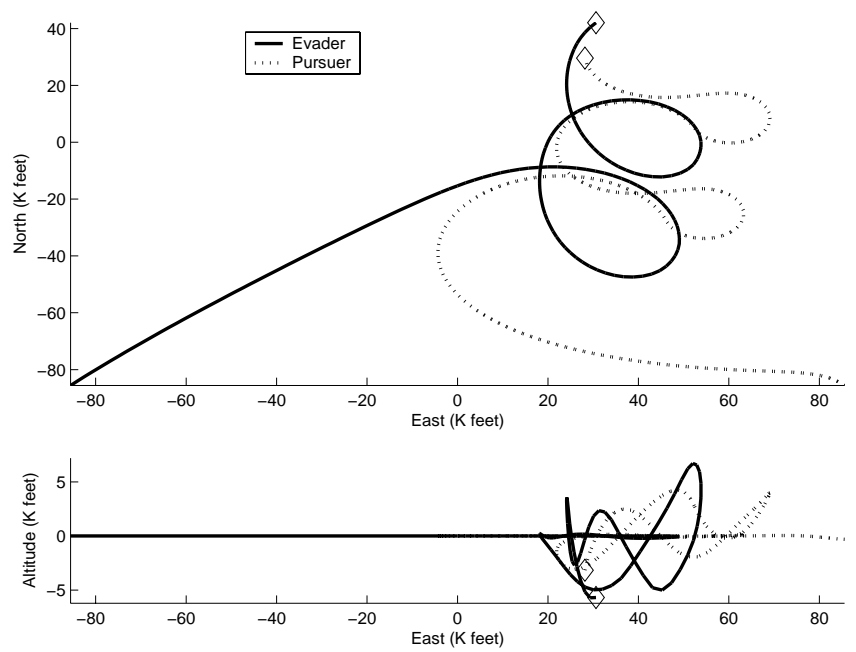

Fig. 5. The same example viewed from above and from the side.

$10^{\circ}$ of directly in front). Fig. 6 shows that the evader has successfully avoided losing the game to this point by staying out of these $10^{\circ} \mathrm{AOT} / \mathrm{AON}$ conditions.

The commands generated by the controller are shown in Fig. 7. These commands are sent to the autopilot in the DemoSim test platform, and will be sent to the T-33 autopilot in the actual test flight.

The behavior of the NMPTC algorithm can be understood by examining individual cost function components that are produced in these maneuvers, and that it is trying to minimize. Fig. 8 shows the trajectory component of the cost function $\left(\tilde{\mathbf{y}}_{k}^{\mathrm{T}} Q \tilde{\mathbf{y}}_{k}\right.$, for $N$ steps, varying by the simulation time), in which the function has a small value while it follows its path to the egress point early in the game, then increases and varies as the evader is forced off the trajectory by the pursuer. In this 


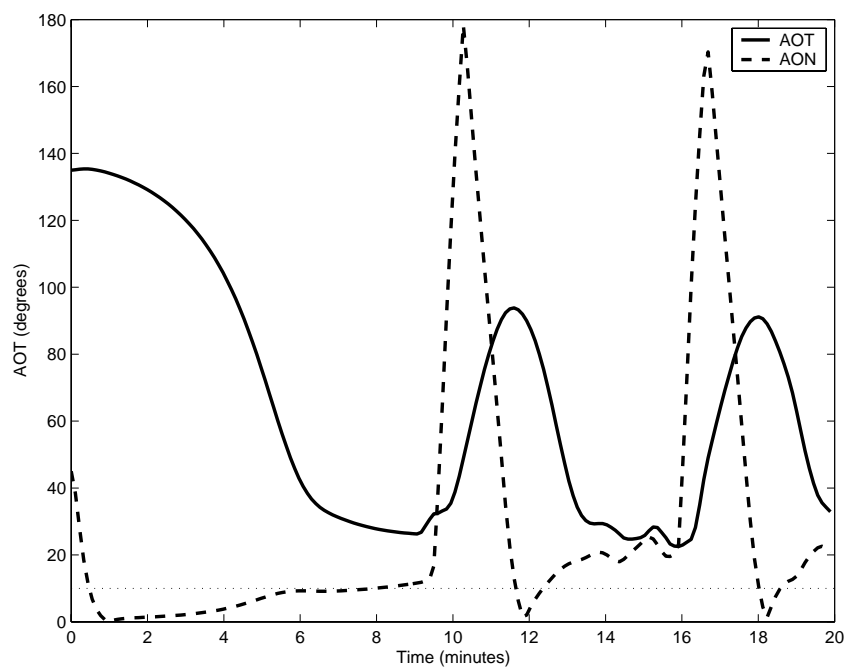

Fig. 6. Win conditions for the pursuit/evasion game (AOT and AON must both be less than $10^{\circ}$ ).
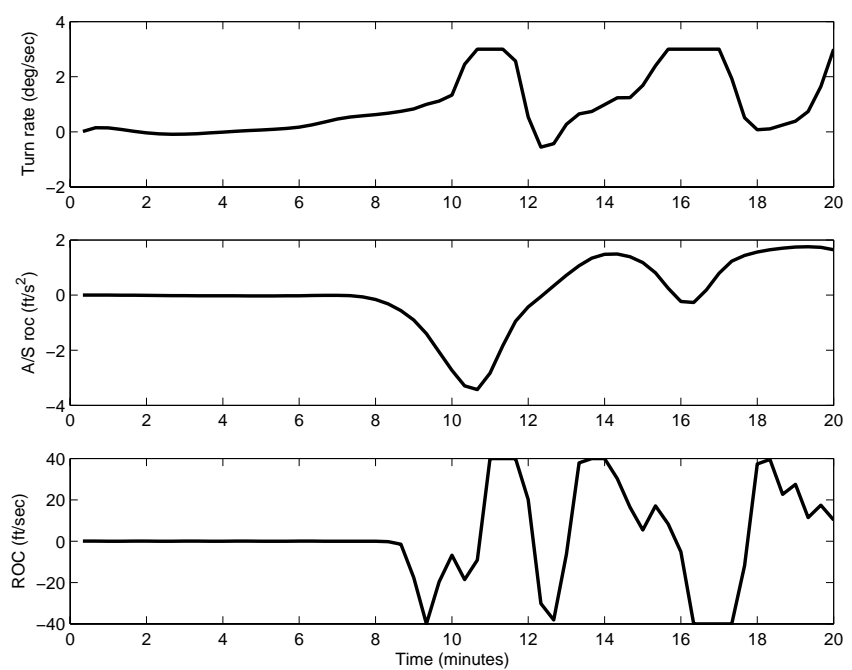

Fig. 7. Autopilot commands generated by the controller, consisting of the turn rate, airspeed rate of change (roc) and rate of climb (ROC).

and the following cost function plots, the predicted cost function calculated at each time step can be seen as slices cut across the "Simulation time" axis. These cost functions use a 30 step look-ahead and the simulations are run for 20 minutes simulation time.

Fig. 9 shows the cost function of the evader's proximity to the pursuer and Fig. 10 shows the separate cost of AOT, which is the cost function that the evader's controller uses to prevent the pursuer from taking a shooting position on its tail.

The constraints on the states of the evader as shown in the cost function in Fig. 11 in which the NMPTC is accounting for limitations in altitude, speed, etc. in its determination of the aircraft's trajectory.

Comparing these figures also allows one to understand

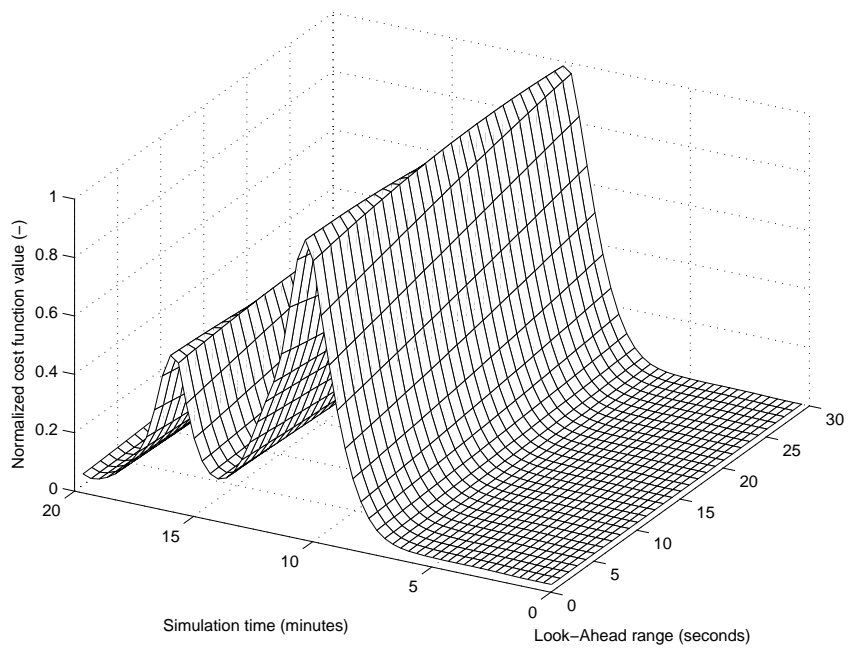

Fio 8 Traiestorv enst function for NMPTC

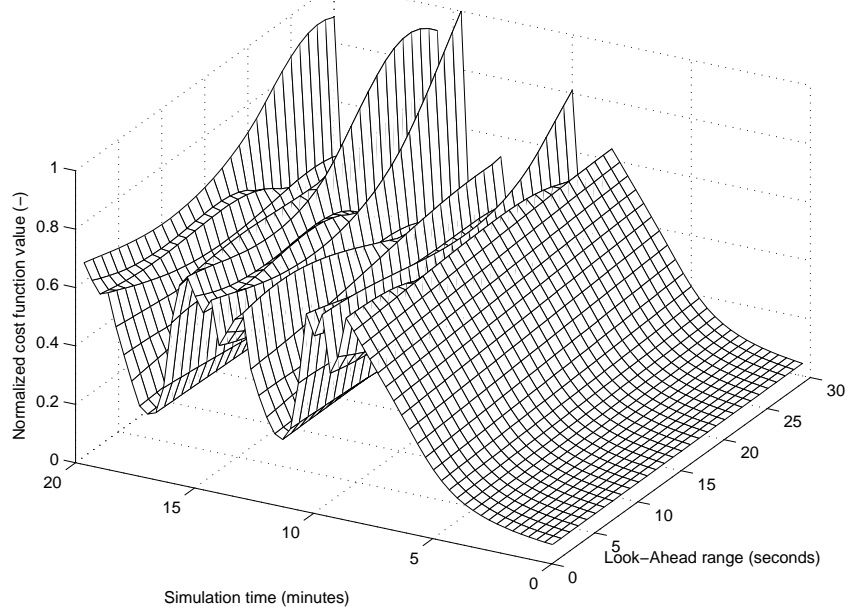

Fig. 9. Evader/pursuer position difference cost function for NMPTC

how the game developed. For example, the value of the proximity penalty was large slightly before 10 minutes (Fig. 9), and this produces large control commands (Fig. 7 ), which prevent the pursuer from getting behind it (Fig. 6). This evasion causes the deviation from the desired trajectory (Fig. 4 and Fig. 5), which increases the trajectory penalty slightly after 10 minutes (Fig. 8). Also, note how that AOT cost function in Fig. 10 increases in the look-ahead around 14 minutes, and how the maneuvers that result successfully prevent this cost from approaching the simulation time axis (i.e. actually happening).

\section{Conclusions and Future Works}

\section{A. Conclusions}

In this paper we have shown the effectiveness of the NMPC approach to the pursuit/evasion game for fixedwing aircraft in a time-critical application. Results from 


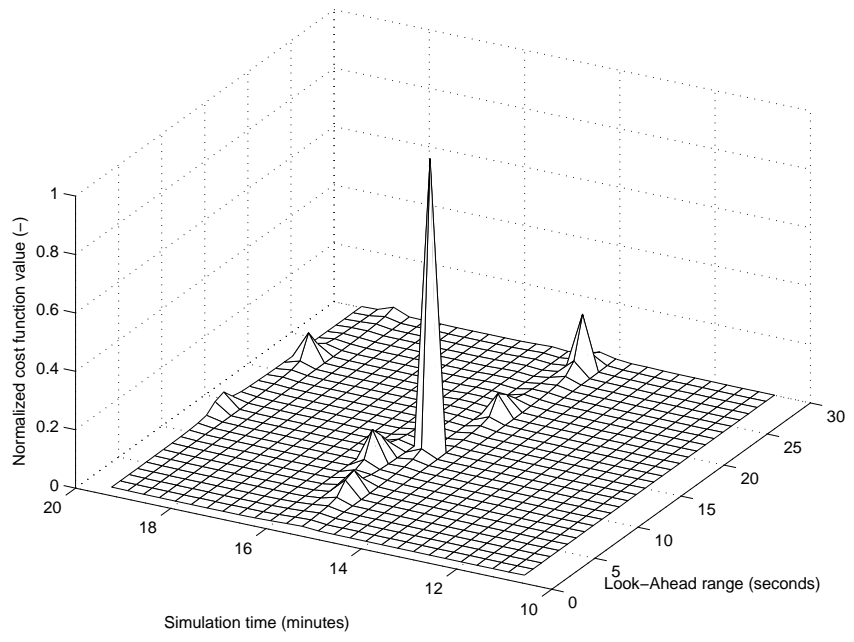

Fig. 10. Anøle off tail (AOT) cost function for NMPTC

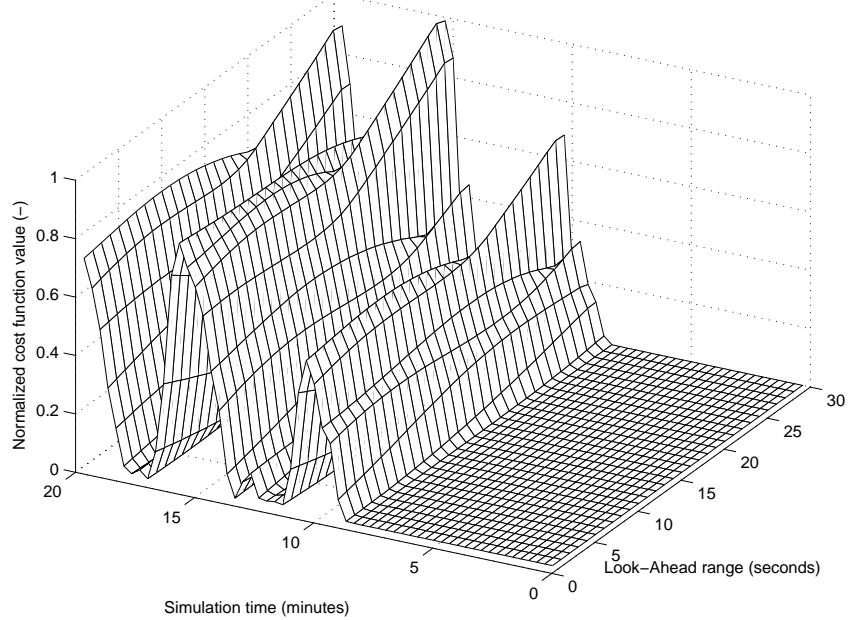

Fig. 11. State cost function for NMPTC

the actual vehicle demonstration will be included with the final paper ${ }^{1}$.

By using the NMPC approach, rapid computations can be performed, and (given accurate dynamics) the true advantages of autonomy can be encoded using the concepts of competitive games. By providing this autonomous mode (e.g., evader) to a UAV operator, it is possible for a remote operator to relinquish control of the vehicle in time-critical situations, to allow the intelligent controller to serve as a surrogate that incorporates the same theories and behaviors of the pilot. Because the safety and functionality constraints of the aircraft are encoded into the cost function, the UAV is not endangering itself or its environment.

\footnotetext{
${ }^{1}$ The demonstration is slated to take place in June of 2004, well after submission time, but before press-time, and will allow ample time to provide feedback from the physical demonstration to evaluate our rapid-simulation approach, as well as justify the use of the DemoSim model, provided to us by the vehicle sponsors.
}

The simulation (and TBD demonstration) results show that the encoding of the game into the cost function was successful. NMPC had not yet been demonstrated on fixed-wing aircraft for the pursuit/evasion problem, and this work shows that this method is appropriate when providing input to an autopilot interface.

\section{B. Future Works}

The application of NMPC will continue as we encode the notion of a symmetric pursuit/evasion game into the cost function. This will enable an aircraft to switch roles in the game, thus switching its goals (and associated costs) at runtime. The encoding of this decision to switch roles as part of the cost-function is an exciting possibility. Also, the use of other strategies (besides the open-loop) will be employed for the live demo, to use a variant of the anticipative strategy where a default strategy of the pursuer is assumed by the evader, despite the absence of the actual input vector values.

Currently the game can only be played with the highoverhead avionics interface to the T-33 jet, provided by Boeing. The execution of the code, however, is extremely fast, and future work will involve the hardware implementation in avionics interface to low-cost fixedwing UAVs.

Proven tactics for evasion and pursuit of fighter aircraft, as discussed in [10], would be useful if encoded into the cost function to encourage these behaviors. Future work into iteratively deriving the weights and values of the cost function matrices could be employed to provide this emergent behavior. Both works are slated for the future, along with simulations that show such behavior emerges from the controller definition.

Finally, the overhead associated with creating a new NMPC controller is substantial and it would be useful to have a high-level understanding of the controller, as well as a way to generate the controller from this higher level. Providing an abstraction for this level of detail is a useful future research area.

\section{ACKNOWLEDGMENTS}

This research funded with the support of the DARPA Software Enabled Control (SEC) program, under contract number DARPA SEC F33615-98-C-3614.

\section{REFERENCES}

[1] AFMC Public Affairs, "Global Hawk UAV supports OEF recon," AFMC News Service Release 1217, December 2002.

[2] H. J. Kim, D. H. Shim, and S. Sastry, "Nonlinear model predictive tracking control for rotorcraft-based unmanned aerial vehicles," in American Control Conference, May 2002.

[3] G. J. Sutton and R. R. Bitmead, Nonlinear Model Predictive Control, ser. Progress in Systems and Control Theory. BaselBoston-Berlin: Birkhäuser Verlag, 2000, vol. 26, ch. Computational Implementation of Nonlinear Predictive Control on a Submarine, pp. 461-471.

[4] T. Başar and G. J. Olsder, Dynamic Non-cooperative Game Theory, 2nd ed. Academic Press, 1995. 
[5] M. Bardi, T. Parthasarathy, and T. E. S. Raghavan, Eds., Stochastic and Differential Games: Theory and Numerical Methods, ser. Annals of International Society of Dynamic Games. Birkhäuser, 1999, vol. 4.

[6] R. Isaacs, Differential Games. John Wiley, 1967.

[7] I. Mitchell, "Application of level set methods to control and reachability problems in continuous and hybrid systems," Ph.D. dissertation, Stanford University, Aug. 2002.

[8] F. Allgöwer and A. Zheng, Eds., Nonlinear Model Predictive Control, ser. Progress in Systems and Control Theory. BaselBoston-Berlin: Birkhäuser Verlag, 2000, vol. 26.

[9] H. J. Kim, D. H. Shim, and S. Sastry, "Decentralized nonlinear model predictive control of multiple flying robots in dynamic environments," in IEEE Conference on Decision and Control, Dec. 2003.

[10] R. L. Shaw, Fighter Combat: Tactics and Maneuvering. Annapolis, Maryland: United States Naval Inst., 1985. 